\title{
Burden and causes of maternal mortality and near-miss in a tertiary care centre of Kerala, India
}

\author{
Sreekumari Umadevi ${ }^{1 *}$, Simi Ayesha ${ }^{1}$, Sreekumari Radha ${ }^{1}$, \\ Anish Thekkumkara Surendran Nair ${ }^{2}$, Krishna Devadhas Sulochana ${ }^{2}$
}

\begin{abstract}
${ }^{1}$ Department of Obstetrics and Gynecology, SAT Hospital, Government Medical College, Trivandrum, Kerala, India
${ }^{2}$ Department of Community Medicine, SAT Hospital, Government Medical College, Trivandrum, Kerala, India
\end{abstract}

Received: 13 January 2017

Revised: 19 January 2017

Accepted: 27 January 2017

\author{
*Correspondence: \\ Dr. Sreekumari Umadevi, \\ E-mail: girishmadhavan@yahoo.com
}

Copyright: () the author(s), publisher and licensee Medip Academy. This is an open-access article distributed under the terms of the Creative Commons Attribution Non-Commercial License, which permits unrestricted non-commercial use, distribution, and reproduction in any medium, provided the original work is properly cited.

\begin{abstract}
Background: Reduction of maternal mortality remains a challenge for developing countries like India as per the sustainable development goals put forward by UN. Near-miss audit is emerging as a new tool for setting new protocols in reduction of MMR. This study aimed, to analyze the near miss events and mortality events which occurred in the study setting from January 2011 to December 2012, and to compare the causes that led to the events.

Methods: A descriptive comparative study was conducted at SAT Hospital, Government Medical College, Thiruvananthapuram, a tertiary care center in Kerala, India on the data accounted for a period of 2 years, 2011 and 2012. Maternal near-miss during the period is studied according to WHO 2009 criteria and compared with the maternal deaths during the same time period. The data was recorded using structured proforma; the same proforma was used to record maternal mortality cases of the same period.

Results: Total live births during the time period were 18,663. Eighty-eight near miss cases and 26 maternal deaths occurred during the study period. Incidence proportion of maternal near-miss was 4.71/1000 live births. Severe maternal outcome was 6 per1000. For every maternal death, there were 3.38 near-misses. Mortality index of our institute was $22.8 \%$ and maternal mortality ratio (MMR) of the study setting was 139/1 Lakh live births. Post-partum hemorrhage was the leading cause for near-miss and systemic diseases were the major contributors to mortality.

Conclusions: It is evident from the present study that PPH, once the leading cause of maternal mortality is now the leading cause of maternal near miss and by improving the resources of FRUs it can be further reduced. Systemic diseases are emerging as a new threat to the obstetric population leading to mortality.
\end{abstract}

Keywords: Mortality index, Maternal mortality, Maternal near-miss, Maternal near miss to mortality ratio, Severe maternal outcome ratio

\section{INTRODUCTION}

Maternal mortality remains a major challenge worldwide in spite of technological advances. Reduction of maternal mortality was one of the eight millennium development goals. The target of MDG 5 was to reduce the MMR by $75 \%$ from 1990 to $2015 .{ }^{1}$ Reduction of maternal mortality to 70 by 2030 is set as goal 3 in sustainable development goals by $\mathrm{UN} .^{2}$

Maternal death is defined as the death of a woman while pregnant or within 42 days of termination of pregnancy or its management irrespective of the site of pregnancy but not from accidental or incidental causes. ${ }^{3}$ 
WHO has setup many guidelines in view of reducing maternal mortality ratio and to improve the quality of care within the health system. It is observed that in recent years the number of maternal deaths in many centers has come down too much that it is no longer meaningful to audit only maternal death. ${ }^{4}$ Review of severe maternal morbidity therefore becomes more relevant and practical. According to the WHO near miss approach for maternal health, in any setting, women who develop severe acute complication during pregnancy share many pathological and circumstantial factors. While some of them die, a proportion of them narrowly escape death. By evaluating these cases with severe maternal outcomes (both near miss cases and maternal deaths) much can be learnt about the processes in place (or lack of them) for the care of pregnant woman. ${ }^{5}$

Maternal near miss refers to a woman who nearly died but survived a complication that occurred during pregnancy childbirth or within 42 days of termination of pregnancy. 6,7

Scrutiny of such cases may be useful for several reasons. Larger numbers of cases could permit more simultaneous reporting. Moreover, lessons learned from the management of cases who survived may be more useful than from these who died, due to the possibility of interviewing the woman herself in near miss cases. Thus, near miss cases can act as controls for deaths and death to severe morbidity ratio reflects the standard of maternal care

For several years, the main impediment to using maternal near miss as a reliable tool for evaluating maternal health conditions was that it was being defined on differing criteria, which led to heterogeneous estimates of its incidence. ${ }^{8,9}$ In 2009, with a view to standardizing the criteria on which maternal near miss is defined, the WHO proposed a new classification using 25 criteria based on the presence of organ and system (cardiovascular, respiratory, renal, hepatic, neurologic, coagulation and uterine) dysfunction. Since then, several studies from various countries and institutions have been published taking these criteria into account. ${ }^{2}$

The maternal mortality ratio (MMR) of India was 254 (2004-2006), which was reduced to 174 (2015). ${ }^{10}$ India is signatory to millennium declaration as well as SDG and is committed to achieving the target of sustainable development goals of reducing MMR to $70 .^{2}$ Even though studies have been done in Rajasthan, Kozhikode (Kerala), India and other parts of the country, with an ongoing confidential review of maternal death in the state of Kerala. ${ }^{7,11,12}$ This study setting, Government medical college Thiruvananthapuram could come out with only a limited number of studies. This study aims to analyse the near miss events and mortality events which occurred in the study setting from January 2011 to December 2012, to compare the causes that led to the events and to analyse the quality of care given to mothers This study also helps to analyse the pattern of referral, what all modification, can be made at FRU level for better patient survival.

\section{METHODS}

Clearance from institutional research committee and institutional ethics committee was obtained before starting the study. The study design was that of a descriptive-comparative study conducted at SAT Hospital, Government Medical college, Thiruvananthapuram Kerala, India from January 2011December 2012. The study setting is one of the largest tertiary care center in south Kerala, SAT Hospital, Thiruvananthapuram where antenatal cases are managed along with referred cases from $100 \mathrm{~km}$ surrounding the hospital. The study participants include all antenatal patients who had severe maternal outcome either maternal death or near miss events.

The definitions for near miss used in this study was the specific criteria given by WHO in $2009 .{ }^{3}$ According to WHO, various events were classified as life threatening conditions present at hospital admissions (Type A) and Life threatening conditions developing during hospital stay after admission (Type B). Various symptoms were classified system wise. Clinical, management and laboratory criteria were identified as per WHO guidelines.

Clinical criteria were acute cyanosis, breathing rate more than 40 or less than 6 , oliguria unresponsive to fluids or diuretics, loss of consciousness for greater than 6 hours, cardiac arrest, jaundice, gasping, shock coagulation disorders, cerebrovascular accident, total paralysis. Laboratory criteria were oxygen saturation $<90 \%$ for $>60 \mathrm{mts}$, acute thrombocytopenia $(<50000)$, creatinine $>3.5 \mathrm{mg} / \mathrm{dl}$, bilirubin $>6 \mathrm{mg} / \mathrm{dl}$, lactate $>5, \mathrm{paO} 2 / \mathrm{FiO} 2$ $<200, \mathrm{pH}<7.1$ Management criteria use of continuous vasoactive drug, dialysis for treatment of acute kidney failure, puerperal hysterectomy due to infection or hemorrhage, cardiopulmonary resuscitation, transfusion greater than 5 units of red blood cell concentrate, intubation and ventilation for a period of $>60 \mathrm{mts}$ unrelated to anesthesia.

In the current study we could identify near misses and subsequently calculate maternal near miss indicators like severe maternal outcome ratio, maternal near miss to mortality ratio and mortality index. Severe maternal outcome ratio refers to the number of women with life threatening condition (MNM+MD) per 1000 live birth. ${ }^{3}$ Maternal near miss ratio refers to the number of maternal near miss cases per 1000 live birth (MNMR= $\mathrm{MNM} / \mathrm{LBx}$ 1000). Both ratios give us an estimation of amount of care and resources needed in an area. Maternal near miss to mortality ratio (MNM: $1 \mathrm{MD}$ ) refers to the ratio between maternal near miss cases and maternal deaths. Higher ratios indicate better care. Mortality index refers to the number of maternal deaths divided by the 
number of women with life threatening condition expressed as a percentage (MI=MD/MNM+MDx100) the higher the index, the more women with life threatening conditions die (low quality of care).

Hospital near miss events were identified by visiting maternal intensive care unit and labour room on daily basis. Data was collected using structured proforma from the time of admission or at a later moment during the patient's stay at the hospital. In addition to review of case sheets, interview of patient (in near misses) and bystander was also done. Information contained socioeconomic background, obstetric score, time of occurrence of complications whether postpartum, intrapartum or antepartum, the type of organ dysfunction, clinical, laboratory and management based criteria was analysed in a particular case along with its neonatal outcome. Before the interview an informed consent was taken regarding the study and its objectives. No alteration in management was made for the sake of the study the number of total live births and maternal mortality in our institute during the study period was also found out. Once data collection was over the information was entered in excel work sheet. The descriptive statistics were analysed using SPSS software. Chi-square test was used to examine difference among the proportions.

\section{RESULTS}

Total live births reported during the study period from the study setting was 18663 . Total women with severe maternal outcome were 114 , out of which 26 maternal deaths occurred. So, maternal mortality ratio of this institute was 139/1lakh live births. Maternal near-miss rate was calculated as 4.71/1000 live births. Severe maternal outcome ratio was six per thousand. For every maternal death 3.38 near-misses occurred. Mortality index of this institute was $22.8 \%$. Mean age (SD) of the study population, women with severe maternal outcome was 26.25 (4.4) years. Majority of cases were belonging to the third decade of life, between 20 and 30 years $(\mathrm{n}=67,76.04 \%)$ of near miss and maternal mortality group $(n=19,73.06 \%)$. Only three $(3.4 \%)$ of near miss and one $(3.8 \%)$ of maternal mortality group belonged to less than 20 years.

Those who were more than 30 years amounted to 18 $(20.45 \%)$ of near miss and six $(23.07 \%)$ of maternal mortality group (Table 1). Most of the study population $(n=8090.9 \%)$ of near miss and mortality group $(n=21$, $80.76 \%$ ) belong to rural areas (Table 1).

Table 1: Baseline socio - demographic characteristics.

\begin{tabular}{|c|c|c|c|c|}
\hline \multirow[t]{2}{*}{ Variable } & \multicolumn{2}{|c|}{ Near miss } & \multicolumn{2}{|c|}{ Maternal death } \\
\hline & $\mathbf{N}=\mathbf{8 8}$ & $\%$ & $\mathrm{~N}=26$ & $\%$ \\
\hline \multicolumn{5}{|l|}{ Age category } \\
\hline$<20$ & 3 & 3.4 & 1 & 3.8 \\
\hline $21-30$ & 67 & 76.04 & 19 & 73.06 \\
\hline$>31$ & 18 & 20.45 & 6 & 23.07 \\
\hline \multicolumn{5}{|l|}{ Place of residence } \\
\hline Rural & 80 & 90.9 & 21 & 80.76 \\
\hline Urban & 8 & 9.09 & 5 & 19.23 \\
\hline \multicolumn{5}{|c|}{ Socio economic status } \\
\hline BPL & 73 & 82.95 & 20 & 76.92 \\
\hline APL & 15 & 17.04 & 6 & 23.07 \\
\hline \multicolumn{5}{|c|}{ Morbidity in relation to obstetric status } \\
\hline First pregnancy & 35 & 39.77 & 11 & 42.3 \\
\hline Second pregnancy & 34 & 38.63 & 7 & 26.92 \\
\hline Third pregnancy & 3 & 3.34 & 2 & 7.6 \\
\hline Forth pregnancy & 0 & 0 & 0 & 0 \\
\hline Five or more & 0 & 0 & 0 & 0 \\
\hline \multicolumn{5}{|l|}{ Mode of delivery } \\
\hline Vaginal & 29 & 32.95 & 11 & 42.3 \\
\hline $\mathrm{CS}$ & 51 & 97.95 & 13 & 60 \\
\hline Hysterectomy & 5 & 5.6 & 0 & 0 \\
\hline Abortion & 2 & 2.27 & 1 & 3.8 \\
\hline MTP & 0 & & 1 & 3.8 \\
\hline Ectopic & 1 & 1.13 & 0 & \\
\hline \multicolumn{5}{|l|}{ Baby details } \\
\hline Live born & 68 & 77.27 & 19 & 73.07 \\
\hline Dead & 14 & 15.9 & 5 & 19.23 \\
\hline
\end{tabular}


Regarding socioeconomic status, $73(82.95 \%)$ of the near miss group and $20(76.92 \%)$ of mortality group belonged to below poverty line. Majority of near miss events occurred in first pregnancy $(n=35,39.77 \%)$. In the mortality group, $11(42.3 \%)$ occurred in the first pregnancy and $7(26.92 \%)$ in the second pregnancy. Coming to higher order pregnancies, the numbers are still less. Caesarean section was the mode of delivery in, 51 $(57.95 \%)$ of near miss events and $13(50 \%)$ of maternal mortality group. Majority of babies in both groups were live born $(n=68,77.27 \%)$ in near miss and $(n=19$, $73.07 \%$ ) in maternal mortality group (Table 1).

Majority of cases $(n=62,70.45 \%)$ of near miss and $(n=20,76.92 \%)$ of mortality group were referred from first referral units. Out of the total 62 referred cases of near miss, $34(54.83 \%)$ was from the different taluk hospitals (higher referral center under public sector), 18 $(29.03 \%)$ was from the different private hospitals, the rest $(\mathrm{n}=10,16 \%)$ from even higher centers like District hospital. Regarding mortality group also majority ( $n=9$, $45 \%$ ) came from Taluk hospitals followed by private hospitals- $(n=6,30 \%)$ and district hospitals $(n=5,25 \%)$. Most common reason for referral was non-availability of adequate blood and blood products and absence of second on call consultant for any surgical intervention to be done-35 (56.45\%), paucity of facilities and manpower for intensive monitoring- 15 (24.19\%), and absence of multidisciplinary team for treatment, especially in case of systemic diseases- $12(19.35 \%)$.

Table 2: Risk factors.

\begin{tabular}{|c|c|c|c|c|c|}
\hline \multirow{2}{*}{ Variable } & \multicolumn{3}{|c|}{ NM $(n=88)$} & \multicolumn{2}{|c|}{ NM $(n=26)$} \\
\hline & $\mathbf{N}$ & $\%$ & $\mathbf{N}$ & $\%$ & $P$ value \\
\hline Hypertensive disorder & 14 & 15.9 & 2 & 7.6 & 0.289 \\
\hline Placenta previa & 12 & 13.6 & 1 & 3.8 & 0.168 \\
\hline Abruption & 5 & 5.6 & 2 & 7.6 & 0.708 \\
\hline Other systemic disease & 11 & 12.5 & 11 & 42.3 & $<0.001$ \\
\hline \multirow{2}{*}{ Post-partum events } & \multicolumn{2}{|c|}{ Near miss $(n=88)$} & \multicolumn{2}{|c|}{$M M(n=26)$} & \\
\hline & $\mathbf{N}$ & $\%$ & $\mathbf{N}$ & $\%$ & \\
\hline PPH & 46 & 52.27 & 4 & 15.38 & 0.004 \\
\hline
\end{tabular}

Time of occurrence of morbidity in near miss was maximum in postpartum period -50 (56.81\%) and postpartum hemorrhage accounted for maximum morbidities- $46(52.27 \%)$. This was found to be statistically significant as post-partum hemorrhage in maternal mortality group was only 4 (15.38\%). Even though the events were postpartum, antepartum risk factors were present in $42(47.72 \%)$ of cases in near miss group whereas it was present in $16(61.53 \%)$ of mortality group. Of the ante partum risk factors, hypertensive disorders ranked first in near miss group- 14 (15.9\%) whereas it accounted for $2(7.6 \%)$ in the mortality group. The second predominant risk factor in near miss was placenta praevia including accrete and percreta 12 $(13.6 \%)$ whereas it was present in one $(3.8 \%)$ of mortality group. In contrast in maternal mortality group in majority of cases- $16(61.53 \%)$ the life-threatening morbidity occurred in the antepartum period and maternal systemic diseases was the major initiating event-11 $(42.30 \%)$. This was found to be statistically significant as life threatening systemic diseases were found in 11 $(12.5 \%)$ of near miss cases. Among systemic diseases, CNS dysfunction was the leading cause- 5 (19.2\%). Systemic diseases occurred either during pregnancy or was a preexisting one worsened by pregnancy (Table 2). Intrapartum morbidities were present in three patients of near miss group only- rupture uterus was seen in one patient $(1.13 \%)$ who was treated with emergency laparotomy followed by hysterectomy and sudden cardiac arrest in 2 patients (2.27\%), both of them could be revived and resuscitated back to life.

Emergency surgical intervention as a life saving measure was needed in $55(62.5 \%)$ of near misses and $4(15.3 \%)$ of mortality group. Emergency laparotomy was done in $13(14.7 \%)$ of near misses, re laparotomy in $10(11.3 \%)$ cases. Obstetric hysterectomy as a life saving measure was performed in $26(29.59 \%)$ of near misses and 1 $(3.8 \%)$ of mortality group.

Hematological dysfunction was the leading organ involvement, accounting for $36(40.90 \%)$ of near miss and $12(46.15 \%)$ of maternal mortality. Within this, DIC amounted to $22(25 \%)$ in near miss and $8(30.76 \%)$ of maternal mortality group. Shock, necessitating use of continuous vasoactive drugs was detected in 34 (38.6\%) of near miss and $4(15.3 \%)$ of the mortality cases. All were treated with timely and massive blood transfusion in case of near miss but in case of maternal mortality group, although vasoactive drug treatment was given, timely blood transfusion was not available which resulted in death of all the four cases-two of them were brought dead while the other two reached this hospital in a moribund state and could not be revived. Blood transfusion was required in $70(79.54 \%)$ of near miss cases. Among these, $38(43.18 \%)$ needed up to five pints' transfusion, 27 $(30.68 \%)$ needed six to ten pints' transfusion and five (5.6\%) needed more than eleven pints. Regarding blood 
component therapy, $61(69.31 \%)$ needed fresh frozen plasma, $33(37.5 \%)$ needed platelet concentrate and 3 (3.4\%) cryoprecipitate. Among those who needed fresh frozen plasma, $45(51.1 \%)$ needed up to five pints, 14 $(15.9 \%)$ needed six to ten pints and $2(2.2 \%)$ needed more than eleven pints. Coming to platelets, $24(27.27 \%)$ needed up to five, $8(9.09 \%)$ required six to ten and 1 $(1.13 \%)$ required more than eleven pints. Ventilator as a life saving measure unrelated to anesthesia was needed for $26(29.54 \%)$ of near misses and 9 (34.61\%) of mortality cases. Dialysis for acute kidney injury was required for $4(4.5 \%)$ of near misses (Table 3$)$.
Table 3: Supportive care given.

\begin{tabular}{|lllll|}
\hline $\begin{array}{l}\text { Supportive care } \\
\text { given }\end{array}$ & $\mathrm{N}$ & $\%$ & $\mathrm{~N}$ & $\%$ \\
\hline Ventilator & 26 & 29.54 & 9 & 32.61 \\
\hline Dialysis & 4 & 4.5 & 0 & 0 \\
\hline ICU stay & & & & \\
\hline$<1$ day & 0 & 0 & 9 & 34.61 \\
\hline $1-4$ & 56 & 63.63 & 4 & 15.38 \\
\hline $5-10$ & 28 & 31.81 & 5 & 19.23 \\
\hline$>10$ & 4 & 4.5 & 4 & 15.38 \\
\hline
\end{tabular}

Table 4: Causes and their mortality indices.

\begin{tabular}{|c|c|c|c|c|c|}
\hline \multirow[t]{2}{*}{ Cause } & \multicolumn{2}{|c|}{ Near miss $(n=88)$} & \multicolumn{2}{|c|}{ MM (n=26) } & \multirow{2}{*}{$\begin{array}{l}\text { Mortality index } \\
\%\end{array}$} \\
\hline & $\mathbf{N}$ & $\%$ & $\mathbf{N}$ & $\%$ & \\
\hline PPH & 46 & $(52.27)$ & 4 & $(15.38)$ & 8 \\
\hline Sepsis & 8 & $(9.09)$ & 2 & (7.6) & 20 \\
\hline Hypertensive disorder & 7 & (7.9) & 2 & (7.6) & 22 \\
\hline CNS dysfunction & 0 & (0) & 5 & (19.2) & 100 \\
\hline Thromboembolism & 0 & & 2 & (7.6) & 100 \\
\hline Renal d dysfunction & 2 & $(2.2)$ & 2 & (7.6) & 50 \\
\hline Liver dysfunction & 2 & $(2.2)$ & 2 & (7.6) & 50 \\
\hline Respiratory dysfunction & 1 & $(1.13)$ & 1 & (3.8) & 50 \\
\hline Cardiac dysfunction & 7 & (7.9) & 2 & (7.6) & 22 \\
\hline Abruption & 5 & $(5.6)$ & 2 & (7.6) & 28 \\
\hline Rupture uterus & 1 & $(1.13)$ & 0 & (0) & 0 \\
\hline Ectopic & 1 & $(1.13)$ & 0 & (0) & 0 \\
\hline ITP & 4 & $(4.5)$ & 0 & (0) & 0 \\
\hline Inversion & 2 & $(2.2)$ & 0 & (0) & 0 \\
\hline
\end{tabular}

When duration of ICU stay was calculated, $56(63.63 \%)$ occupied ICU for up to four days, 28 (31.81\%) for 5-10 days and $4(4.54 \%)$ for more than ten days (Table 3$)$. Out of the four who had prolonged ICU stay two had sepsis, one had PPH following LSCS, which was managed by relaparotomy and ventilatory support, another one was a case of heart disease with valve replacement done.

PPH was the leading cause in near-miss 46 (52.27\%) whereas it came to $4(15.38 \%)$ in the mortality group with a mortality index of $8 \%$. Sepsis amounted to 8 $(9.09 \%)$, in near miss and $2(7.6 \%)$ of mortality group (mortality index of $20 \%$ ). The third common cause was hypertensive disorders $7(7.9 \%)$ in near miss and 2 (7.6\%) of maternal deaths (mortality index-22\%) (Table 4).

CNS dysfunction $(n=5,19.2 \%)$ was the leading cause of maternal mortality and no such cases were identified in near miss amounting to a mortality index of $100 \%$. Thrombo embolism constituted $2(7.6 \%)$ of maternal mortality and no such cases identified in near miss leading to a mortality index of $100 \%$. Causes of CNS dysfunction were refractory epilepsy (two cases), CVA, intra cerebral bleed (one case), sub arachnoid bleed in an APLA +ve BOH patient (one case), meningo encephalitis (one case). Among the other causes liver and renal failure had a mortality index of $50 \%$ (Table 4).

\section{DISCUSSION}

The near miss incidence rate in the present study is 4.71 per 1000 live births which agrees with studies conducted in Rajasthan in India (4.18/1000LB). ${ }^{11}$ This is also similar to other studies conducted in Sri Lanka $(5.5 / 1000)$, meta-analysis in USA $(0.42 \%)$, Brazil $(4.4 / 1000)$ and the WHO systematic review $(0.4 \%$ $8 \%) .{ }^{17,18,22,25}$ The rates were higher in Rwanda $(21.5 / 1000 \mathrm{LB})$, Kathmandu (23/1000)..$^{14,16}$

The wide variation in results is due to different criteria used for identifying near miss events This study strictly adhered to the WHO 2009 criteria leading less number of near miss events. Near miss rates are generally found higher in resource poor settings. ${ }^{18} \mathrm{WHO}$ has put forward a new elaborate inclusion criteria in 2014 in order to pick up all mothers with potential life threatening 
complications, the study results of which are yet to come out.

Maternal near miss to mortality ratio (3.38:1) in present study is closer to the rate in Barpeta (3.9:1) Rajasthan (2.07:1) Ahmedabad (2.58:1) South Africa (5-8), but higher rates are observed in Europe (117-223), Scotland (49). ${ }^{11,19,23,26-28}$ Higher rates indicate better quality of care.

MMR in this institute was 139 which agrees with that of, Kozhikode (153.5) in Kerala and MMR of India 178 but lower than Rajasthan- 202, Barpetta- 1085, Rwanda-325, Ahmedabad- 832.8. ${ }^{11,12,14,19,23,29}$ This does not match with MMR in Kerala which was 66 in 2016

Majority of near miss cases were referred from FRUs and hence this does not reflect the mortality rate of the patients directly registered in this institution and the denominator should include live births from all the FRUs. SMOR in this study was $6 / 1000$ which agrees with that of Sri Lanka (5.8/1000). ${ }^{17}$

Hematological dysfunction amounted to $34.09 \%$ of cases in near miss and $34.61 \%$ of mortality cases which was the leading organ dysfunction. DIC amounted to $25 \%$ of near miss events and $30.76 \%$ of mortality cases. Prompt replacement of blood and blood products in case of obstetric hemorrhage and early identification and treatment of sepsis before DIC sets in, are the preventive options.

Mortality index of this institute was $22.8 \%$, which agrees with that of Barpetta- 20.45\% and Ahmedabad- 27.8\% but higher when compared to Sri Lanka- 5.2\% and Scotland (2\%). ${ }^{17,19,23,28}$ Lower rates indicate better care.

PPH was the leading initiating event in near misses $(52.27 \%)$ it's mortality index was low (8\%) indicating good quality care. This agrees with many Indian studies and that of Rwanda. ${ }^{11,14-16,19,21}$ Sepsis was the second leading cause $(9.09 \%)$ with a mortality index of $20 \%$. Similar finding is seen in Rwanda. ${ }^{14}$ This is an alarming situation probably due to emergence of antibiotic resistant organisms and failure to recognize signs of infection earlier and changing over to higher antibiotics. The third leading cause was hypertensive disorders $(7.9 \%)$ which also had relatively high mortality index of $22 \%$. Most of these cases were referred late. Strict maternal surveillance with more frequent BP check-ups and urine examination for albuminuria have to be strictly adhered to for early detection of pre-eclampsia. These three are the leading causes of near miss in some of the Indian studies also, ${ }^{11,12,15}$ and one study from Rwanda. ${ }^{14}$ Thromboembolism accounted for $2(7.6 \%)$ of maternal deaths while no such cases occurred in near miss group accounting to a mortality index of $100 \%$. This finding is also very alarming as thromboembolism is fatal once occurred and strategies like prophylactic heparinisation for high risk patients should be made a hospital protocol.
Organ dysfunction leading to severe maternal outcome like liver dysfunction, renal dysfunction and respiratory dysfunction etc. although the actual number were less carried high mortality rates. ${ }^{23}$

\section{CONCLUSION}

This finding suggests to advocate strict screening protocols from the first antenatal visit itself to identify them and to initiate correct treatment modalities for maternal systemic diseases in conjunction with appropriate specialists as early as possible. A multidisciplinary team should be set alert in tertiary care centers in order to initiate treatment without delay once such cases are admitted.

Funding: No funding sources

Conflict of interest: None declared

Ethical approval: The study was approved by the Institutional Ethics Committee

\section{REFERENCES}

1. UN Millennium Project. Available from. http://www.unmillenniumproject.org/goals/ Accessed 07 Jan 2017.

2. UN Sustainable Development Goals. Available from http://www.un.org/sustainabledevelopment/health/ Accessed 07 Jan 2017.

3. World Health Organization. International statistical classification of diseases and related health problems. Availabe from http://www.who.int/ classifications/ icd/ en/

4. Paily VP, Ambujam K, Rajasekharan Nair V, Thomas B. Confidential review of maternal deaths in Kerala: a country case study. BJOG: Int J Obstet Gynecol. 2014;1;121(4):61-6.

5. World Health Organization. Evaluating the quality of care for severe pregnancy complications: the WHO near-miss approach for maternal health. Geneva: World Health Organization. 2011:29.

6. Prual A, Bouvier- Colle Bernis L, Breat G. Severe maternal morbidity from direct obstetric causes in West Africa: incidence and case fatality rates. Bull World Health Organization. 2000;78:593-602.

7. Report on the World Health Organization Working Group on the Classification of Maternal Deaths and Severe Maternal Morbidities. Geneva, World Health Organization, 2009. Available from http:// apps.who.int/ iris /bitstream/ 10665/ 44692/ 1/9789241502221_eng.pdf. Accessed 07 Jan 2017.

8. Say L, Souza JP, Pattinson RC. Maternal Near-Misstowards a standard tool for monitoring quality of maternal health care. Best Pract Res Clin Obstet Gynecol. 2009;30; 23(3):287-96.

9. Lobato G, Nakamura-Pereira M, Mendes-Silva W, Dias MA, Reichenheim ME. Comparing different diagnostic approaches to severe maternal morbidity and near-miss: a pilot study in a Brazilian tertiary 
hospital. Eur J Obstet Gynecol Reprod Biol. 2013;167:24-8.

10. Maternal mortality ratio in India 2010. Available from: http:/ /www.unicef.org/ infobycountry/ india_statistics.html. Accessed 07 Jan 2017.

11. Kalra P, Kachhwaha CP. Obstetric Near-Miss morbidity and maternal mortality in a tertiary care centre in Western Rajasthan. Indian J Public Health. 2014;58(3):199.

12. Chandran JR, Raj SV, Sumangala Devi D, Ramachandran A. Maternal Near-Miss review from a tertiary care center in South India. Int J Reprod Contracept Obstet Gynecol. 2016;5(8):2657-60.

13. Purandare C, Bhardwaj A, Malhotra M, Bhushan H, and Chhabra S, Shivkumar P. Maternal near-miss reviews: lessons from a pilot programme in India. BJOG: Int J Obstet Gynecol. 2014; 121(4):105-11.

14. Kalisa R, Rulisa S, van den Akker T, van Roosmalen J. Maternal Near-Miss and quality of care in a rural Rwandan hospital. BMC pregnancy and childbirth. 2016 Oct 21; 16(1):324.

15. Taly A, Gupta S, Jain N. Maternal intensive care and 'Near-Miss' mortality in obstetrics. J Obstet Gynecol India. 2004;54(5):478-82.

16. Shrestha NS, Saha R, Karki C. Near-Miss maternal morbidity and maternal mortality at Kathmandu Medical College Teaching Hospital. Kathmandu University Medical Journal. 2010;8(2):222-6.

17. Ranatunga G, Akbar J, Samarathunga S, Perera Y, Kariyawasam L, Kumarasiri J. Severe acute maternal morbidity in a tertiary care institution. Sri Lanka J Obstet Gynecol. 2013;34(4):85-8.

18. Tunçalp Ö, Hindin MJ, Souza JP, Chou D, Say L. The prevalence of maternal Near-Miss: a systematic review. BJOG: Int J Obstet Gynecol. 2012; 119(6):653-61.

19. Sarma HK, Sarma HK, Kalita AK. A prospective study of maternal near-miss and maternal mortality cases in FAAMCH, Barpeta; with special reference to its aetiology and management: first 4 months report. J Obstet Gynaecol Barpeta. 2015;1(2)100-6.

20. Bindal J, Solanki G. Clinical and etiological study of maternal near-miss at a Tertiary Referral Hospital of Central India. Indian $\mathrm{J}$ Obstet Gynecol Res. 2016;3(1):28-31.
21. Jahan S, Begum K, Shaheen N, Khandokar M. NearMiss/Severe acute maternal morbidity (SAMM): A new concept in maternal care. J Bangladesh Coll Phys Surg. 2006;24(1):29-33.

22. Lotufo FA, Parpinelli MA. Haddad SM, Surita FG, Cecatti JG. Applying the new concept of maternal near-miss in an intensive care unit. Clinics. 2012;67(3):225,

23. Sahijwani DV, Desai A, Kansara V. Analysis of Near-Miss cases as a reflection of emergency. D Gynaecology. 2013;5(3):99-101.

24. Mhyre JM, Bateman BT, Leffert LR. Influence of patient comorbidities on the risk of near-miss maternal morbidity or mortality. Journal American Soc Anesthesiologist. 2011;115(5):963-72.

25. Say L, Pattinson RC, Gülmezoglu AM. WHO systematic review of maternal morbidity and mortality: the prevalence of severe acute maternal morbidity (near miss). Reproductive health. 2004;1(1):1.

26. Prual A, Huguet D, Garbin O, Rabe G. Severe obstetric morbidity of the third trimester, delivery and early puerperium in Niamey (Niger). African $\mathrm{J}$ Reprod Health. 1998;2(1):10-9.

27. Waterstone M, Murphy JD, Bewley S, Wolfe C. Incidence and predictors of severe obstetric morbidity: case-control study. Commentary: Obstetric morbidity data and the need to evaluate thromboembolic disease. BMJ. 2001;322(7294):1089-94.

28. Brace V, Penney G, and Hall M. Quantifying severe maternal morbidity: a Scottish population study. BJOG: Int J Obstet Gynecol. 2004;111(5):481-4.

29. Pathak D, Chakraborty B, Goswami S, Adhikari S. Changing trends of maternal mortality: a comparative study. J Obstet Gynecol India. 2011;61(2):161-5.

Cite this article as: Umadevi $S$, Ayesha $S$, Radha $S$, Nair ATS, Sulochana KD. Burden and causes of maternal mortality and near-miss in a tertiary care centre of Kerala, India. Int J Reprod Contracept Obstet Gynecol 2017;6:807-13. 